\title{
Características de la neumonía por Pneumocystis jiroveci en adultos con SIDA con y sin terapia antiretroviral
}

\author{
Laura Bahamondes M., M. José Villar Z., Carolina Orellana C., Jimena González R. y Cristian Montenegro U.
}

\section{Pneumocystis jiroveci pneumonia characteristics in adults with AIDS with or without antiretroviral therapy}

Highly active antiretroviral therapy (HAART) has changed the epidemiology of Pneumocystis jiroveci pneumonia (PCP) in AIDS patients. Global incidence of PCP has decreased and now it is prevalent in AIDS patients who do not receive HAART or are unsuccessfully treated with persistent immune depression. Moreover, the immunologic response to HAART has caused a PCP form which is included in the immune restoration inflammatory syndrome (IRIS). As of late 2004, 75.5\% of patients cared for at Dr. Lucio Córdova Infectious Diseases Hospital were receiving HAART. This study compares PCP clinical characteristics in patients under the effect of HAART (n: 6) with those without antiretroviral therapy (n: 12). Among those with HAART, $83.3 \%$ (5/6) were without immunologic responses and $16.7 \%$ with virologic response. The median CD4 counts were low in both groups: 20 cells $/ \mathrm{mm}^{3}$ without HAART and 51 cells $/ \mathrm{mm}^{3}$ with HAART. There were no differences in most of PCP characteristics, and no IRIS cases were observed. HAART-receiving group had less severe disease and lower frequency of both, complications and steroidal therapy prescription (P 0.023).

Key words: Pneumocystis jiroveci, pneumonia, AIDS, PCP, HAART.

Palabras claves: Pneumocystis jiroveci, SIDA, TARV.

\section{Introducción}

$\mathrm{L}$ a epidemiología de la neumonía por Pneumocystis jiroveci (NPj), así denominada hoy la especie infectante específica del hombre ${ }^{1}$, ha cambiado con posterioridad a la introducción de las terapias antiretrovirales (TARV) de alta actividad, en el tratamiento de los pacientes con SIDA.

La incidencia de esta infección oportunista disminuye precozmente en los primeros tres meses de $\mathrm{TARV}^{2}$, como consecuencia de la restauración inmune, expresada entre otros cambios, en un ascenso en el recuento de linfocitos CD4.

En la era post TARV, los casos nuevos de NPj corresponden principalmente a pacientes con SIDA sin TARV, o con fracaso de esta terapia. En los países industrializados, la incidencia descendió de 4,9 casos/ 100 persona-año antes de marzo de 1995 , año en que comenzó en ellos el uso masivo de TARV, a 0,3 casos/ 100 personas-año después de marzo de $1998^{3}$.

En el Hospital de Enfermedades Infecciosas Dr. Lucio Córdova (HEIDLC), como el resto de los hospitales públicos de Chile, el uso expandido de TARV se inició en el año 2001, y a fines del 2004, el 75,5\% (n: 238 pacientes) en control ya recibían TARV.
En este período se observó un descenso en la importancia relativa de $\mathrm{NPj}$ como causa de hospitalización, de $11 \%$ en el año 2001 a $5 \%$ en el 2003. Así también, en la cohorte chilena de pacientes con SIDA del grupo SidaChile, la prevalencia de NPj era 20,5\% antes del inicio de TARV, y la incidencia en los pacientes con SIDA en TARV descendió a $1,2 \%{ }^{4}$.

Junto a estos cambios en la epidemiología, se ha generado una nueva forma de presentación de la NPj en los pacientes que inician TARV. Se trata de un transitorio deterioro clínico, radiológico y gasométrico cuando simultáneamente con esta complicación infecciosa se inicia la TARV ${ }^{5}$. Este cuadro, incluido en lo que se ha denominado sindrome inflamatorio por restauración inmune, o reacciones inflamatorias por restauración inmune ${ }^{6,7}$, se atribuye a la recuperación de respuesta inmune contra antígenos de $P$. jiroveci.

El objetivo de este estudio transversal y de observación es conocer las características de la NPj en los pacientes con SIDA, con y sin TARV.

\section{Pacientes y Método}

Se revisó la historia clínica de 24 pacientes con SIDA egresados del HEIDLC con diagnóstico de NPj,
Hospital de Enfermedades Infecciosas Dr. Lucio Córdova Santiago, Chile (LBM, CMU)

Universidad de Santiago Santiago, Chile Estudiante de Medicina (MJVZ, (OC, JGR).

Recibido: 15 mayo 2005 Aceptado: 12 febrero 2006

\section{Correspondencia a:} Laura Bahamondes M. bahamondeslaura @hotmail.com 
entre enero del año 2002 y julio del 2004. Fueron criterios de inclusión al estudio:

- Caso con confirmación microbiológica: presencia de $P$. jiroveci en muestra respiratoria de lavado broncoalveolar (LBA), expectoración espontánea o expectoración inducida, por método de tinción de Grocott o IFD.

- Caso sin confirmación microbiológica: pacientes con infección por VIH con cuadro clínico característico y sin otra causa confirmada, con hallazgos radiológicos habituales en $\mathrm{NPj}^{8}$ y ascenso de la enzima LDH en sangre más de 2 veces el valor normal $(>2 \mathrm{VN})$.

El recuento de linfocitos CD4 se midió por técnica de citometría de flujo y la carga viral de VIH por técnica NASBA (NucliSens, bioMèrieux).

\section{Definiciones:}

- Forma grave de NPj al ingreso: cuadro con alteración gasométrica expresada en $\mathrm{PO}_{2}<70 \mathrm{mmHg}$ o saturación de $\mathrm{O}_{2}<90 \%$ o diferencia A-a $>35 \mathrm{mmHg}^{8}$.

- Fracaso de la terapia específica de NPj: persistencia de fiebre y/o deterioro gasométrico o radiológico cumplido el quinto día de terapia adecuada, y habiendo descartado otra causa asociada?

- Alergia a cotrimoxazol oral: presencia de rash, con o sin fiebre, posterior al inicio del fármaco, y que cede con la suspensión del mismo.

- Restauración inmune: ascenso de CD4 en al menos 50 céls $/ \mathrm{mm}^{3}$ en el control al cuarto mes de TARV y de al menos 80 céls $/ \mathrm{mm}^{3}$ en control a 12 meses de TARV $^{10}$.

- Éxito virológico del TARV: carga viral de VIH $<80$ copias ARN/ml (límite de detección del examen) o descenso en 2 logs desde la basal en los primeros 4 a 6 meses de TARV.

Análisis estadístico. Para el análisis de comparación de proporciones de las variables categóricas y cuantitativas discretas se aplicó el test $\chi^{2}$ con $\mathrm{p}$ significativo $<0,05$.

\section{Resultados}

En 18 de 24 pacientes analizados se cumplieron los criterios de inclusión al estudio. En 27,7\% (5/18), existió confirmación microbiológica de $\mathrm{PNj}$, y los 13 restantes cumplieron los criterios no microbiológicos.

Terapia anti-retroviral. Al momento del episodio de NPj, 12 pacientes no recibían TARV (grupo sin TARV) y 6 (grupo con TARV) habían iniciado esta terapia (Tabla 1).
En el grupo con TARV, la infección por VIH se había confirmado entre 1 mes y 4 años antes de la NPj, con una mediana de 11,5 meses. El esquema de TARV en estos 6 pacientes incluía zidovudina + lamivudina (Combivir $\left.{ }^{\circledR}-\mathrm{COM}\right)+$ indinavir en $1, \mathrm{COM}+$ abacavir $(\mathrm{ABC})$ en 2, $\mathrm{COM}+$ nevirapina (NVP) en 2 y lamivudina $(3 \mathrm{TC})+$ stavudina $(\mathrm{d} 4 \mathrm{~T})+\mathrm{ABC}$ en 1 paciente.

Estado virológico de la infección por VIH. La mediana de carga viral de VIH al momento de la NPj fue similar en ambos grupos, 65.000 copias ARN/ml (log 4,60) (3.500 - 540.000 copias ARN/ml) en el grupo sin TARV, y 75.000 copias ARN/ml $(\log 4,70)(<\mathrm{LDE}-$ 960.000 copias ARN/ml) en el grupo con TARV. El éxito virológico del TARV se había alcanzado en un paciente.

Estado inmunológico. En el grupo sin TARV, al momento de la NPj la mediana de CD4 fue 20 céls $/ \mathrm{mm}^{3}$, $\left(12-106\right.$ céls $\left./ \mathrm{mm}^{3}\right)$. En el grupo con TARV, la mediana de CD4 fue 51 céls $/ \mathrm{mm}^{3}$ (15 - 360 céls $/ \mathrm{mm}^{3}$ ). Sólo uno de los 6 pacientes en TARV presentó restauración inmune adecuada al momento de la $\mathrm{PNj}$, con ascenso de CD4 a 360 céls $/ \mathrm{mm}^{3}$. En los 5 restantes, el ascenso de CD4 fue $<80$ céls $/ \mathrm{mm}^{3}$ con más de 4 meses de TARV, y 15 céls $/ \mathrm{mm}^{3}$ en el paciente con un mes de TARV. La frecuencia de CD4 $<80$ céls $/ \mathrm{mm}^{3}$ al momento de la NPj fue similar en ambos grupos, 83,3\% (10/12) en el grupo sin TARV y $83,5 \%(5 / 6)$ en el grupo en TARV.

Profilaxis de NPj. Todos los pacientes con TARV y recuento de CD $4<200 / \mathrm{mm}^{3}$, cumplían profilaxis primaria con cotrimoxazol oral $960 \mathrm{mg}$ en días alternos, con buena adherencia (Tabla 1). Ninguno de los pacientes sin TARV recibió profilaxis primaria de $\mathrm{NPj}$, ya que ésta representó el debut clínico de la infección por VIH.

Edad y sexo. En el grupo sin TARV, la mediana de edad fue de 40 años y en el grupo con TARV 33 años. En ambos hubo predominio masculino, 11 hombres en el grupo sin TARV y 6 hombres en el grupo con TARV.

Confirmación etiológica. En la mitad de los pacientes (9/18), existió estudio de muestra de secreción respiratoria: 2 de LBA, 5 de expectoración espontánea y 2 de expectoración inducida con solución salina hipertónica. En 6 muestras se realizó tinción de Grocott, con resultado positivo en 3 , y en las 3 muestras restantes se realizó IFD con resultado positivo en 2 de ellos; en total se confirmó la presencia de $P$. jiroveci en 5 de 9 muestras respiratorias $(55,5 \%)$.

En 10 de $12(83,3 \%)$ pacientes sin TARV, la infección por VIH se diagnosticó junto con la NPj, y fue el evento definitorio de SIDA. En los 2 restantes, la condición de infección por VIH era conocida por el paciente desde 5 y 7 años antes, respectivamente, permaneciendo sin control médico por propia voluntad. En ellos también la $\mathrm{PNj}$ fue el evento definitorio de SIDA.

Todos los pacientes presentaron fiebre y tos, seca 
en $61,1 \%(11 / 18)$ y escasamente productiva en $38,8 \%$ (7/18). En todos, la radiografía de tórax evidenció infiltrados intersticiales en ambos campos. La frecuencia de $\mathrm{LDH}>2 \mathrm{VN}$ al ingreso fue similar en ambos grupos, $81,8 \%(9 / 11)$ en el grupo sin TARV y $60,0 \%$ (3/5) del grupo con TARV (p 0,175) (Tabla 1).

Gravedad del cuadro al ingreso. La forma grave de $\mathrm{PNj}$ fue más frecuente en el grupo sin TARV, alcanzando a 50,0\% (6/12), en tanto que en el grupo con TARV esta frecuencia fue $16,7 \%(1 / 6)$, diferencia no significativa (p 0,086).

Tratamiento anti-infeccioso. Todos los pacientes iniciaron cotrimoxazol (15 mg/kg/día de trimetoprim), administrado vía oral cada 8 horas en 13 casos, e intravenoso cada 6 horas los 5 primeros días de terapia en 5 de 6 pacientes con forma severa de $\mathrm{NPj}$, con switch posterior a terapia oral. En 3 pacientes debió reemplazarse el cotrimoxazol por la asociación clindamicina + primaquina, por fracaso terapéutico (n: 2), uno en el grupo sin TARV $u$ otro en el grupo con TARV (p 0,322), o por alergia a cotrimoxazol y fracaso de la des-sensibilización (n: 1). En los dos casos de fracaso terapéutico se cambió a clindamicina (inicialmente intravenosa) en dosis $600 \mathrm{mgr}$ cada 6 horas (y switch a terapia oral), + primaquina $15 \mathrm{mgr} /$ día oral. Igual esquema antimicrobiano recibió el paciente en quien fracasó la des-sensibilización con cotrimoxazol. Todos completaron 21 días de terapia antimicrobiana y mantuvieron profilaxis secundaria con cotrimoxazol, o dapsona en los casos tratados con la alternativa clindamicina más primaquina.

Corticoterapia. En los 7 pacientes que ingresaron con una forma grave de $\mathrm{NPj}$, se indicó corticoterapia desde el inicio con dexametasona intravenosa en dosis de 4 mgrs cada 8 horas los 5 primeros días en 6 pacientes, pasando luego a prednisona oral $1 \mathrm{mgr} / \mathrm{kg} /$ día, los días 6 a 10 y dosis decreciente los últimos 11 días, para completar un total de 21 días. En un paciente se empleó prednisona desde el inicio en dosis de 1,5 $\mathrm{mg} / \mathrm{kg} /$ día, continuando con el esquema antes mencionado, hasta enterar 21 días. Durante la evolución, hubo deterioro gasométrico en $25,0 \%$ (3/12) del grupo sin TARV mientras que en el grupo con TARV esto ocurrió en $17 \%(1 / 6)$, la diferencia no es significativa (p 0,086). En estos pacientes se indicó corticoterapia en el esquema antes mencionado. Se prescribió corticoterapia con mayor frecuencia en el grupo sin TARV que en el con TARV, 8/12 $(66,6 \%)$ versus $1 / 6$ $(16,7 \%)$, respectivamente $(\mathrm{p}<0,023)$.

La fiebre $>37,5^{\circ} \mathrm{C}$ axilar persistente por más de 5 días durante la terapia con cotrimoxazol oral también fue de frecuencia similar en ambos grupos, 25,0\% (3/12) en el grupo sin TARV y $16,7 \%(1 / 6)$ en el grupo con TARV (p 0,344).

\begin{tabular}{|c|c|c|c|}
\hline Variables analizadas & $\begin{array}{c}\text { Pacientes sin } \\
\text { TARV } \\
\text { (n: 12) }\end{array}$ & $\begin{array}{l}\text { Pacientes } \\
\text { con TARV } \\
\text { (n: 6) }\end{array}$ & $\mathbf{p}$ \\
\hline Edad años (mediana) & 40 & 33 & \\
\hline Sexo $M / F$ & $11 / 12$ & $6 / 6$ & \\
\hline LT CD4 céls/mm³ (mediana) & 20 & 51 & \\
\hline Carga viral copias ARN/ml (mediana) & 65.000 & 75.000 & \\
\hline Log carga viral VIH (mediana) & 4,60 & 4,70 & \\
\hline Pacientes con restauración inmune & * & $1 / 6$ & \\
\hline Pacientes con éxito virológico TARV & * & $1 / 6$ & \\
\hline Profilaxis de $P$. jiroveci & $0 / 12$ & $6 / 6$ & \\
\hline $\mathrm{PO}_{2} \mathrm{mmHg}$ al ingreso (mediana) & 61 & 78 & \\
\hline Pacientes con LDH > 2VN (\%) & $9 / 11(81,8)$ & $3 / 5(60,0)$ & 0,175 \\
\hline CD4 < 80 céls $/ \mathrm{mm}^{3}$ (\%) & $10 / 12(83,3)$ & $5 / 6(83,5)$ & \\
\hline Pacientes con saturación $\mathrm{O}_{2}<90(\%)$ & $2 / 8(25,0)$ & $1 / 6(16,7)$ & 0,353 \\
\hline Pacientes con $\mathrm{PO}_{2}<70$ mmHg (\%) & $6 / 12(50)$ & $1 / 6(16,7)$ & 0,086 \\
\hline Fiebre $>37,5^{\circ} \mathrm{C}$ ax $>5$ días CTX (\%) & $3 / 12(25)$ & $1 / 6(16,7)$ & 0,344 \\
\hline Pacientes con deterioro gasométrico (\%) & $3 / 12(25)$ & $0 / 6$ & 0,090 \\
\hline Fracaso terapéutico & $1 / 11(9)$ & $1 / 6(16,7)$ & 0,322 \\
\hline Alergia a cotrimoxazol & $2 / 12(16,7)$ & $0 / 6$ & 0,144 \\
\hline Des-sensibilización exitosa & $1 / 2(50)$ & * & \\
\hline Corticoterapia & $8 / 12 \quad(66,7)$ & $1 / 6(16,7)$ & 0,023 \\
\hline Ventilación mecánica & $0 / 12$ & $0 / 6$ & \\
\hline Letalidad & $0 / 12$ & $0 / 6$ & \\
\hline
\end{tabular}

Reacciones adversas. Reacción alérgica a cotrimoxazol oral presentaron 2 pacientes del grupo sin TARV $(16,7 \%)$ y ninguno del grupo con TARV (p 0,144). Estos 2 pacientes fueron sometidos a des-sensibilización con cotrimoxazol oral en dosis crecientes, resultando exitosa en un paciente, y en el otro se debió cambiar terapia a clindamicina más primaquina según el esquema antes mencionado.

No fue necesaria la ventilación mecánica ni hubo letalidad en ambos grupos.

\section{Discusión}

Las características observadas en estos pacientes concuerdan con el patrón epidemiológico descrito para la NPj en la era post TARV, es decir, la mayor parte eran pacientes sin TARV. En el grupo en TARV, predominaron los pacientes con fracaso virológico y el 
éxito de terapia alcanza sólo a 16,7\%, comparado con el éxito del TARV en el total de la cohorte chilena que oscila entre 46,5 y $69,9 \%{ }^{4}$.

En el grupo de pacientes que recibían profilaxis con cotrimoxazol oral, la NPj se presentó con recuentos de CD4 inferiores a 200 céls $/ \mathrm{mm}^{3},\left(\right.$ mediana 51 céls $\left./ \mathrm{mm}^{3}\right)$, situación que se describe en la literatura, recordando que en pacientes con inmunodepresión tan severa, la $\mathrm{NPj}$ puede presentarse pese a la profilaxis con cotrimoxazol'.

La confirmación microbiológica de la NPj en este grupo fue baja $(27,7 \%, 5 / 18)$, a pesar que se utilizó IFD, excelente método para detección de $P$. jiroveci en muestra respiratoria ${ }^{11}$. Esto se explica por la dificultad para obtener una muestra respiratoria en esta neumopatía en que el compromiso pulmonar es de predominio intersticial, existiendo tos no productiva en más de la mitad de los casos. En este grupo, 61,1\% $(11 / 18)$ presentó tos seca, lo que impidió obtener muestra respiratoria, excepto en 2 pacientes en que se realizó broncoscopia. El análisis de estos casos, motivó que durante el año 2004, se adquiriera un nebulizador ultrasónico y se implementara la técnica de expectoración inducida con solución salina hipertónica administrada por nebulización ultrasónica. Se ha demostrado que, en la muestra respiratoria obtenida por este procedimiento, la IFD tiene sensibilidad hasta $90 \%$, acercándose al rendimiento de la muestra de LBA que oscila entre 90 y $100 \%{ }^{12}$. Si bien la técnica de RPC alcanza a 96 - 100\% de sensibilidad, la especificidad es menor y oscila entre 59 y $90 \%$ en distintas publicaciones $^{12,13}$. Esta gran variabilidad se atribuye a diferencias en la técnica aplicada, interferencia por ADN polimerasas, o presencia de ADN del huésped y ADN de bacterias en las muestras (especialmente las muestras obtenidas con procedimientos no invasores $)^{14}$.

La ausencia de recuperación inmune en todos, excepto uno de los pacientes en esta serie, explica que no presentaran el cuadro de tipo inflamatorio por res- tauración inmune, el que se expresa en pacientes con TARV por un deterioro clínico y gasométrico. Por el contrario, la indicación de corticoterapia, ya sea por severidad o complicaciones de la NPj, fue menor en el grupo en TARV $(\mathrm{P}<0,023)$ y el único paciente con restauración importante de la inmunidad al momento de la PNj (CD4 de 360 céls $/ \mathrm{mm}^{3}$ ), evolucionó favorablemente. En la literatura es también escasa la frecuencia con que $P$. jiroveci se asocia a enfermedad inflamatoria por restauración inmune, predominando en citomegalovirus y micobacterias (Mycobacterium tuberculosis y especies atípicas $)^{15-17}$. Se mantiene sin embargo, la recomendación de expertos para diferir el inicio de TARV hasta completar la terapia de la NPj y evitar el riesgo de presentación de este cuadro ${ }^{18}$.

\section{Resumen}

La terapia antiretroviral (TARV) de alta actividad cambió la epidemiología de la neumonía por Pneumocystis jiroveci $(\mathrm{NPj})$ en pacientes con SIDA. La incidencia global ha descendido y ahora prevalece en pacientes sin TARV o con fracaso de ésta. Además, la restauración inmune por TARV genera una forma de $\mathrm{NPj}$ incluida en los síndromes inflamatorios por restauración inmune (SIRI). A fines del 2004, 75,5\% de pacientes con infección por VIH en control en el Hospital de Enfermedades Infecciosas Dr. Lucio Córdova tenían TARV. Esta serie describe las características clínicas de la NPj, comparando el grupo con TARV (n: 6) y sin TARV (n: 12). De aquellos con TARV, 83,3\% (5/6) estaban con fracaso inmunológico y $16,7 \%$ (1/6) en éxito virológico. El recuento de CD4 fue bajo en ambos grupos (mediana 20 céls $/ \mathrm{mm}^{3}$ sin TARV y 51 céls $/ \mathrm{mm}^{3}$ con TARV). No hubo diferencia en la mayoría de las características de la $\mathrm{NPj}$, tampoco casos de SIRI. El grupo con TARV tuvo menos severidad y complicaciones, y menos indicación de corticoterapia (p 0,023).

\section{Referencias}

1.- Sidhu G S, Cassai N D, Pei Z. Pneumocystis carinii: an update. Ultrastruct Pathol 2003; 27: 115-22. Review.

2.- Ledergerber B, Egger M, Erard V, Weber R, Hirschel B, Furrer H, et al. AIDS-related opportunistic illnesses occurring after initiation of potent antiretroviral therapy: the Swiss HIV Cohort Study. JAMA 1999; 282: 2220-6.

3.- Morris A, Lundgren J D, Masur H,
Walzer P D, Hanson D L, Frederick T, et al. Current epidemiology of Pneumocystis pneumonia. Emerg Infect Dis 2004; 10: 1713-20.

4.- Beltrán C, Wolff M, GRUPO SIDA CHILE. Datos de la cohorte nacional. www.sidachile.cl (Accedido 2 abril 2005).

5.- Dean G L, Williams D I, Churchill D R, Fisher M J. Transient clinical deterioration in HIV patients with Pneumocystis carinii pneumonia after starting highly active antiretroviral therapy: Another case of immune restoration inflammatory syndrome. Am J Respir Crit Care Med 2002; 165: 1670.

6.- Desimone J A, Pomerantz R J, Babinchak T J. Inflammatory reactions in HIV-1-infected persons after initiation of highly active antiretroviral therapy. Ann Intern Med 2000; 133: 447-54.

7.- Martyn A H. Immune restoration disease in HIV-infected patients on HAART. AIDS Read 1999; 9: 548-9, 554-5, 559-62.

8.- Levine E. Diagnosing pulmonary infections 
in HIV-positive patients. Infect Med 1999; 16: 802-14.

9.- Smego R A, Nagar S, Maloba B, Popara M. A meta-analysis of salvage therapy for Pneumocystis carinii pneumonia. Arch Intern Med 2001; 161: 1529-33.

10.- Al-Harthi L, Voris J, Patterson B K, Becker S, Eron J, Smith K Y, et al. Evaluation of the impact of highly active antiretroviral therapy on immune recovery in antiretroviral naive patients. HIV Med 2004; 5: 55-65.

11.- Olsson M, Stralin K, Holmberg H. Clinical significance of nested polymerase chain reaction and immunofluorescence for detection of Pneumocystis carinii pneumonia. Clin Microbiol Infect 2001; 7: 492-7.
12.- González J, Rubio R, Antela A, García F. III Reunión anual GESIDA. Palmas de Mallorca, 1998. Avances en el diagnóstico, profilaxis y tratamiento de la neumonía por Pneumocystis carinii en pacientes VIH +. www. gesidaseimc.com. (Accedido 22 marzo 2005).

13.- Pinlaor S, Mootsikapun $P$, Pinlaor $P$, Phunmanee A, Pipitgool V, Sithithaworn P, et al. PCR diagnosis of Pneumocystis carinii on sputum and bronchoalveolar lavage samples in immunocompromised patients. $\mathrm{J}$ Parasitol Res 2004; 94: 213-8.

14.- Torres A. Diagnóstico de la NPC en pacientes con infección por VIH: PCR - $P$. carinii versus tinciones convencionales. 5th CROI 1998. www.gesidaseimc.com. (Accedido 10 junio 2004).
15.- Race E M, Adelson-Mitty J, Kriegel G R, Barlam T, Reimann K Letvin N, et al. Focal mycobacterial lymphadenitis following initiation of protease-inhibitor therapy in patients with advanced HIV-1 disease. Lancet 1998; 351: 252-5.

16.- Furrer H, Malinverni R. Systemic inflammatory reaction after starting highly active antiretroviral therapy in AIDS patients treated for extrapulmonary tuberculosis. Am J Med 1999; 106: 371-2.

17.- John M, French M A. Exacerbation of the inflammatory response to Mycobacterium tuberculosis after antiretroviral therapy. Med J Aust 1998; 169: 473-4.

18.- Davaro R, Himlan P. Immune restoration disorders following HAART. AIDS Reader 1999; 9: 167-9. 